\title{
New Measurement Method for Thermal Contact Resistance by Periodic Heating Method Using Lock-In Thermography
}

\author{
by T. Ishizaki*, T. Igami*, A. Ueno*, H. Nagano*
}

*Affiliation 1, Nagoya University, Department of Mechanical System Engineering, Chikusa-Furo, 464-8603, AichiNagoya, Japan, ishizaki.takuya@f.mbox.nagoya-u.ac.jp

\begin{abstract}
This paper proposes a new thermal contact resistance measurement method using lock-in thermography. By the lock-in thermography with an infrared microscope, the temperature distribution across the contact interface was visualized in the sample side surface. Meanwhile, a new thermal contact resistance measurement principle was derived by the superimposition of the temperature wave from virtual heat sources in consideration of the thermal contact resistance at the interface. Consequently, the thermal contact resistance was obtained as a fitting parameter by fitting the theoretical curve to the experimental amplitude and phase lag. the validity of the principle was shown.
\end{abstract}

\section{Introduction}

The thermal contact resistance (TCR) is a phenomenon that the heat flow is impeded at the contact interface between two materials. Reduction of TCR is the issue for discharging heat waste efficiently. In terms of TCR measurement, the steady state method [1] is generally used to measure the overall TCR of the bulk sample, additionally as unsteady methods, the thermo reflectance [2] method and the $3 \omega$ method [3] are studied to measure TCR of the homogenous thin film sample. However, the method to measure the local TCR in the contact interface is not established. This paper proposes a new local TCR measurement method by a periodic heating method. Besides, the validity of the method is confirmed. The temperature wave behavior at the contact interface was visualized using a lock-in thermography (LIT) with an infrared microscope. Furthermore, TCR was estimated by fitting the theoretical curve of the amplitude and the phase lag of the temperature wave to the measurement plots.

\section{Method and Measurement}

The measurement method is discussed on the two layers system consisted of two thin plates $A$ and $B$ bonded together. When the edge of the A layer surface is heated periodically by a laser focused in a spot, the temperature wave from the heat source reflects and transmits repeatedly at the sample surface and the contact interface. Then, temperature response is expressed as Eqs. (1) and (2) by the method of mirror images. In the method of mirror images temperature response is obtained by the superimposition of the temperature wave from the virtual heat sources in consideration of the thermal contact resistance at the interface.

$$
\begin{aligned}
& \text { When } n=2 m+1(m=0,1,2,3, \ldots) \\
& \begin{array}{l}
\left\{\begin{array} { l } 
{ T _ { A } ( r , t ) = [ \sum _ { n = 0 } ^ { \infty } \frac { T _ { 0 } } { ( n + 1 ) d - r } \mathrm { e } ^ { - \mu _ { A } \{ ( n + 1 ) d - r \} } G _ { A _ { n } } ] \mathrm { e } ^ { i \omega t } } \\
{ T _ { B } ( r , t ) = [ \sum _ { n = 0 } ^ { \infty } \frac { T _ { 0 } } { ( n - 1 ) d + r } \mathrm { e } ^ { - \mu _ { B } \{ ( n - 1 ) d + r \} } G _ { B _ { n } } ] \mathrm { e } ^ { i \omega t } }
\end{array} \quad \text { where, } \left\{\begin{array}{l}
G_{A_{n}}=G_{A_{n-1}} \gamma(n d)+G_{B_{n-1}} T(n d) \\
G_{B_{n}}=G_{A_{n-1}} T(n d)+G_{B_{n-1}} \gamma(n d)
\end{array}\right.\right. \\
\text { When } n=2(m=0,1,2,3, \ldots)
\end{array} \\
& \left\{\begin{array} { l } 
{ T _ { A } ( r , t ) = [ \sum _ { n = 0 } ^ { \infty } \frac { T _ { 0 } } { n d + r } e ^ { - \mu _ { A } \{ n d + r \} } G _ { A _ { n } } ] \mathrm { e } ^ { i \omega t } } \\
{ T _ { B } ( r , t ) = [ \sum _ { n = 0 } ^ { \infty } \frac { T _ { 0 } } { ( n + 2 ) d - r } \mathrm { e } ^ { - \mu _ { B } \{ ( n + 2 ) d - r \} } G _ { B _ { n } } ] \mathrm { e } ^ { i \omega t } }
\end{array} \text { where, } \left\{\begin{array}{ll}
G_{A_{0}}=1, & G_{A_{n}}=G_{A_{n-1}} \\
G_{B_{0}}=0, & G_{B_{n}}=G_{B_{n-1}}
\end{array}\right.\right.
\end{aligned}
$$

$T$ is the temperature, the subscribe $A, B$ represents layer $A$ and $B, r$ is the distance in thickness direction, $t$ is time, $T_{0}$ is the temperature normalization constant, $n$ is the number of the reflectance and transmittance, $d$ is the thickness, $\mu=(1+i) \sqrt{\omega / 2 D}, \omega$ is the angular frequency, $D$ is the thermal diffusivity, $\gamma$ and $t$ are the reflectance and transmittance coefficient at contact interface with TCR. The $y$ and $\tau$ at the surface and the back surface are 1 and 0 respectively by the assumption of the insulation. From Eqs. (1) and (2), the amplitude and the phase lag of the temperature wave along $r$ from the heating point is obtained. At the contact interface $(r=d)$, the amplitude and the phase lag jump depending on only TCR when the thermal diffusivity of the A and B layer is known. Therefore, TCR is obtained by fitting the theoretical amplitude and phase lag to the plots measured by LIT. The measurement apparatus is consisted of a diode laser (638 $\mathrm{nm}$ ), an objective lens to focus the laser beam in a spot, LIT device with the infrared microscope, a function generator to oscillate periodic signal, a PC to conduct lock-in analysis. A sample was made by the bonding and lamination for two isotropic graphite IG-110 plate (thickness is $0.48 \mathrm{~mm}$ ). The total thickness of the prepared sample was $0.965 \mathrm{~mm}$. The thermal 
conductivity and the thermal diffusivity of IG-110 are $126 \mathrm{~W} /(\mathrm{m} \cdot \mathrm{K})$ and $102 \times 10^{-6} \mathrm{~m}^{2} / \mathrm{s}$ [5]. TCR measurements were conducted in the conditions of $1 \mathrm{~Hz}$ and $20 \mathrm{~Hz}$, to confirm the influence of the frequency to the results.

\section{Discussion}

The contour of the figure 1 shows the amplitude and the phase lag distribution obtained by lock-in analysis in the $1 \mathrm{~Hz}$ heating measurement. It is confirmed that the amplitude and the phase lag have discontinuity and jumps at the contact interface. Incidentally, the distribution above the sample surface indicate the temperature response of the atmosphere excited by the sample heating. The plots and curve of figure 1 show the fitted theoretical curve and the measurement plots. Although the curve and plots show good agreement, the difference becomes greater near the heat source. This is caused by the difference of the heat source size between the measurement theory and the actual apparatus That is, the diameter of the actual heat source is finite, while theoretical heat source is a spot. However, this difference can be negligible according to being away from the heat source. Table 1 lists the results of the fitting analysis of the amplitude and the phase lag in $1 \mathrm{~Hz}$ and $20 \mathrm{~Hz}$ heating.
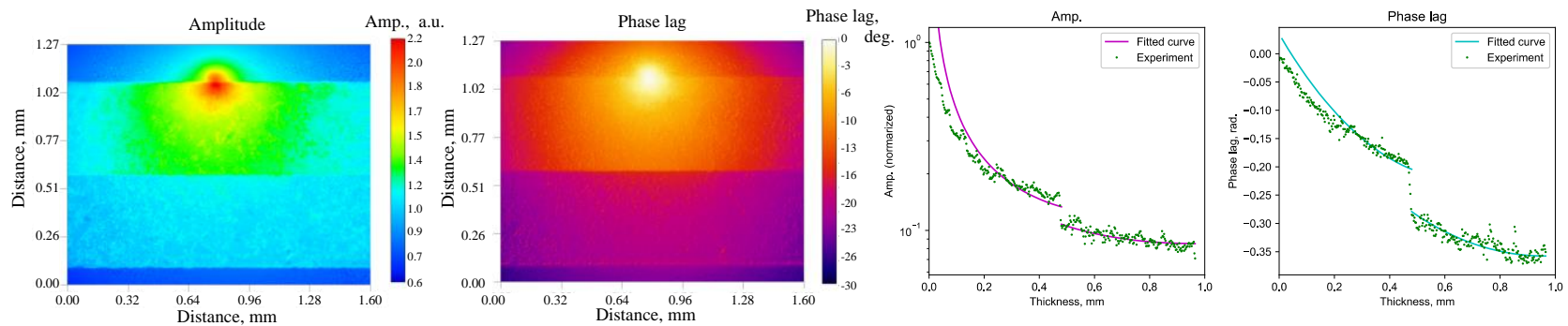

Fig. 1: Amplitude and phase lag distribution and fitting analysis for TCR in $1 \mathrm{~Hz}$ heating.

Table 1. Results of fitting analysis of amplitude and phase lag in $1 \mathrm{~Hz}$ and $20 \mathrm{~Hz}$ heating.

\begin{tabular}{|l|l|l|l|}
\hline TCR, $\mathrm{m}^{2} \mathrm{~K} / \mathrm{W}$ & Amplitude fitting & Phase lag fitting & Difference \\
\hline $1 \mathrm{~Hz}$ heating & $1.60 \times 10^{-6}$ & $2.33 \times 10^{-6}$ & $46 \%$ \\
\hline $20 \mathrm{~Hz}$ heating & $2.37 \times 10^{-6}$ & $2.81 \times 10^{-6}$ & $19 \%$ \\
\hline Difference & $48 \%$ & $21 \%$ & \\
\hline
\end{tabular}

Table 1 shows the good agreement of the TCR on $10^{-6} \mathrm{~m}^{2} \mathrm{~K} / \mathrm{W}$ order among measurement conditions of the amplitude fitting, the phase lag fitting and frequencies. However, the difference of the results is greater in the amplitude fitting than the phase lag one, because the amplitude is more susceptible for the difference of the heat source size discussed before. Besides, the difference of the results is greater in the $1 \mathrm{~Hz}$ measurement than $20 \mathrm{~Hz}$ one, because the lower frequency measurement is more susceptible for the difference of the heat source due to becoming thermally close to the heat source for its longer thermal diffusion length.

\section{Conclusion}

A new TCR measurement method was proposed. First, the periodic heating measurement theory derived by the method of mirror images was shown. Then, using the LIT device with infrared microscope, the behavior of the amplitude and the phase lag of the temperature wave throw the contact interface was observed. Consequently, it was confirmed that the amplitude and the phase lag jump at the contact interface. Finally, TCR was evaluated by the fitting analysis of the theoretical amplitude and phase lag and measurement ones. Hence, the validity of the measurement was confirmed.

\section{Acknowledgements}

A part of this research was supported by consignment research expenses (JPMJCR12I2) from JST CREST.

\section{REFERENCES}

[1] Y. Xian et al., Appli. Thermal Eng., 130, (2018), 1530-1548.

[2] A. Moridi et al., Surf. Coat. Tech., 334, (2018), 233-242.

[3] D. G. Cahill et al., J. Heat Trans., 124(2), (2002), 223-241.

[4] M. Bertolotti et al., J. Appli. Phys., 85(7), (1999), 3540-3545.

[5] NMIJ, Certified Reference Materials Catalog 2017-2018, Retrieved April 14, 2019, n.d., https://www.nmij.jp/service/C/CRM_Catalog_(JE)_2017-2018.pdf. 